\title{
Event shapes at hadron colliders
}

\author{
Andrea Banfi* ${ }^{\dagger}$ \\ ETH Zurich, Institute for Theoretical Physics \\ E-mail: banfieitp.phys.ethz.ch
}

We present precision results for distributions in global event shapes that can be measured at hadron colliders within experimental limitations. These predictions are obtained by combining exact next-to-leading order (NLO) with the all-order resummation of large logarithms of soft and collinear origin. We then discuss how event-shape measurements can be used for the tuning of Monte Carlo event generators, for tests of models of hadronisation and underlying event, and as discriminatory tools between QCD jet-like and New Physics events.

XVIII International Workshop on Deep-Inelastic Scattering and Related Subjects, DIS 2010 April 19-23, 2010

Firenze, Italy

\footnotetext{
* Speaker.

${ }^{\dagger}$ This work has been done in collaboration with Gavin Salam (LPTHE Jussieu, Paris) and Giulia Zanderighi (Oxford University).
} 


\section{Introduction}

Event-shape variables are infrared and collinear safe measures of the geometrical properties of the hadronic energy momentum flow, giving an idea on whether an event is pencil-like, planar, spherical, etc. Measurements of their mean values and distributions have played a crucial role at LEP, for precise determinations of the strong coupling $\alpha_{s}$, for tests of analytical models of hadronisation corrections, and for validation of Monte Carlo (MC) event generators (see [1] for a recent review). In spite of the success of these studies in $e^{+} e^{-}$annihilation, very little attention has been devoted to their counterparts at hadron colliders $[2,3]$. This was mainly because the quantities that were conveniently measured experimentally could not be accurately computed in perturbative QCD. Here I would like to present event shapes that can be measured at actual hadron colliders, and whose distributions can be computed in perturbative QCD at the accuracy needed to have a reliable estimate of the associated theoretical uncertainties.

\section{Event-shape variables at hadron colliders}

In hadron-hadron collisions, we consider events with two hard central jets, and define event shapes that vanish in the limit of two narrow jets. For instance, given all hadrons $\left\{q_{i}\right\}$ in a rapidity region $\mathscr{C}$ (for instance $\left|\eta_{i}\right|<\eta_{\mathscr{C}}$ ) and using transverse momenta $\vec{q}_{\perp, i}$ only, we define the transverse thrust $T_{\perp}$ and the thrust minor $T_{m}$ as follows

$$
T_{\perp}=\max _{\vec{n}_{\perp}} \frac{\sum_{i \in \mathscr{C}}\left|\vec{q}_{\perp, i} \cdot \vec{n}_{\perp}\right|}{\sum_{i \in \mathscr{C}}\left|\vec{q}_{\perp, i}\right|}, \quad T_{m}=\frac{\sum_{i \in \mathscr{C}}\left|\vec{q}_{\perp, i} \times \vec{n}_{\perp}\right|}{\sum_{i \in \mathscr{C}}\left|\vec{q}_{\perp, i}\right|} .
$$

We can also introduce boost-invariant event shapes involving longitudinal degrees of freedom, like invariant masses or broadenings, or the three-jet resolution parameter $y_{3}$. An extensive list of hadronic event-shape definitions can be found in $[4,5]$.

There are three basic reasons why dijet event shapes can be studied experimentally with very first data. First, cross sections for dijet production are large both at the Tevatron and at the LHC, as shown in table 1. From table 1 one can also see that the flavour content of a sample can be varied by changing the leading jet $p_{t}$-cut. Low- $p_{t}$ samples (Tevatron with $p_{t 1}>50 \mathrm{GeV}, \mathrm{LHC}$ with $p_{t 1}>200 \mathrm{GeV}$ ) are gluon dominated, while initial-state quarks become more important for high- $p_{t}$ samples (Tevatron with $p_{t 1}>200 \mathrm{GeV}$, LHC with $p_{t 1}>1 \mathrm{TeV}$ ). Second, event shapes are

\begin{tabular}{|l|cc|ccc|}
\hline & LO & NLO & $q q \rightarrow q q$ & $q g \rightarrow q g$ & $g g \rightarrow g g$ \\
\hline Tevatron, $p_{t 1}>50 \mathrm{GeV}$ & $60 \mathrm{nb}$ & $116 \mathrm{nb}$ & $10 \%$ & $43 \%$ & $45 \%$ \\
Tevatron, $p_{t 1}>200 \mathrm{GeV}$ & $59 \mathrm{pb}$ & $101 \mathrm{pb}$ & $41 \%$ & $43 \%$ & $12 \%$ \\
$14 \mathrm{TeV}$ LHC, $p_{t 1}>200 \mathrm{GeV}$ & $13.3 \mathrm{nb}$ & $23.8 \mathrm{nb}$ & $7 \%$ & $40 \%$ & $50 \%$ \\
$14 \mathrm{TeV}$ LHC, $p_{t 1}>1 \mathrm{TeV}$ & $6.4 \mathrm{pb}$ & $10.5 \mathrm{pb}$ & $31 \%$ & $51 \%$ & $17 \%$ \\
\hline
\end{tabular}

Table 1: Cross sections for the production of two jets in a central rapidity region $\left(\left|y_{\text {jets }}\right|<0.7\right.$ at the Tevatron and $\left|y_{\text {jets }}\right|<1$ at the LHC) with cut on $p_{t 1}$, the transverse momentum of the leading jet. On the right it is possible to see the relative importance of each partonic subprocess.

normalised quantities: experimental uncertainties associated with jet energy scale cancel between numerators and denominators, see eq. (2.1). Finally, since one usually measures normalised differential distributions, like $1 / \sigma d \sigma / d T_{m}$, no determination of luminosity is required. 
From a theoretical point of view, event-shape distributions can be computed at NLO with NLOJET++ [6]. However, as can be seen in the example plots of figure 1, both LO and NLO predictions diverge at small values of $T_{m, g}$ (the directly global variant of $T_{m}$, see section 3 ). Only a combination of NLO and next-to-leading logarithmic (NLL) resummation (labelled NLL+NLO) gives a distribution that is sensible for any value of $T_{m, g}$. In particular, resummation restores the correct physical behaviour at $T_{m, g} \rightarrow 0$, corresponding to vanishing probability of having accelerated charges without accompanying radiation.

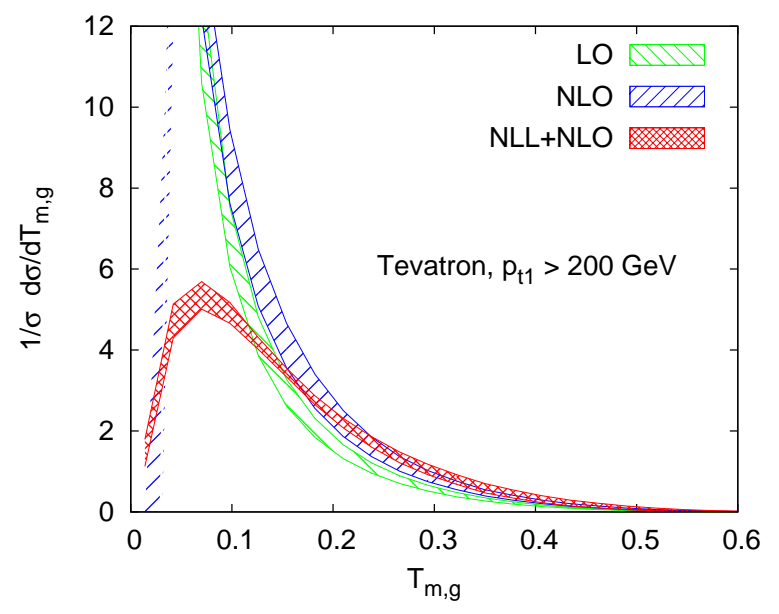

Figure 1: The differential distribution in $T_{m, g}$ for a Tevatron high- $p_{t}$ sample. The bands represent theoretical uncertainties.
NLL resummation involves writing the integrated $T_{m, g}$ distribution as an exponent $\Sigma\left(T_{m, g}\right)=\exp \left[L_{1}\left(\alpha_{s} L\right)+g_{2}\left(\alpha_{s} L\right)\right]$, with $L=\ln \left(1 / T_{m, g}\right), g_{1}\left(\alpha_{s} L\right)$ resumming the leading logarithms $\left(\mathrm{LL}, \alpha_{s}^{n} L^{n+1}\right)$, and $g_{2}\left(\alpha_{s} L\right)$ the NLL, $\alpha_{s}^{n} L^{n}$. Knowledge of $g_{1}\left(\alpha_{s} L\right)$ determines the position of the peak of the differential distribution, typically in the region $\alpha_{s} L \sim 1$. In the peak region $g_{2}\left(\alpha_{s} L\right)$ becomes of order one, and is therefore needed to stabilise both the position and the height of the peak.

NLL resummability is guaranteed for variables satisfying the following conditions [7]: a specific functional dependence on a single soft and collinear emission; (continuous) globalness, i.e. sensitivity to emissions in the whole of the phase space; recursive infrared and collinear (rIRC) safety, a subtle mathematical condition on the event-shape scaling properties with multiple emissions. If these conditions are satisfied, the relevant emissions that contribute to event-shape distributions at NLL accuracy are soft and collinear parton clusters widely separated in rapidity, which, due to QCD coherence, can be considered as independently emitted from hard legs. Since a similar pattern of emissions is simulated by MC event generators, one may expect that most features of rIRC safe global event-shape distributions are correctly described by these theoretical tools.

Of the three constraints, the most difficult to satisfy experimentally is globalness, due to the fact that the measurement region $\mathscr{C}$ is preferably restricted to the central detector region (e.g. $|\eta| \lesssim$ 2.5 at the LHC), and in any case no measurement is actually performed in the very forward regions (corresponding to a limiting rapidity $\eta_{c}=5$ at the LHC). However [4], one can devise classes of global event shapes even at hadron colliders: directly global, where the region $\mathscr{C}$ extends up to the maximum available rapidity $\eta_{c}$; exponentially suppressed, where $\mathscr{C}$ is inside the acceptance of the central detectors (e.g. $|\eta|<1$ at the Tevatron and $|\eta|<1.5$ at the LHC), while outside this region we add to the event-shape definition a term that exponentially suppresses the contribution of hadrons in the forward regions; recoil, where measurements are performed only in a central region $\mathscr{C}$, and we add a term that is sensitive to emissions outside $\mathscr{C}$ through recoil. In the last case however a numerical breakdown of NLL resummation occurs in the region where the event shape is small. 


\section{Event-shape distributions the Tevatron and at the LHC}

In [5] we have performed a NLL+NLO resummation for a number of selected event shapes. Figure 2 shows predictions for the directly global thrust minor $T_{m, g}$, together with theoretical uncertainties. The latter, aimed at having an indication of missing NNLO and NNLL corrections, are estimated via: asymmetric variation of renormalisation and factorisation scales $\mu_{R}$ and $\mu_{F}$; variation of the logarithm to be resummed $\ln \left(X T_{m, g}\right)$ with $1 / 2<$ $X<2$; variation of the matching scheme (log-R or mod-R). Similar results are obtained for all considered event shapes, and show that uncertainties are under control and within $\pm 20 \%$ in a wide range of values of $T_{m, g}$.

These predictions are valid at parton level only, so it is interesting to investigate the impact of hadronisation and underlying event on eventshape distributions. This can be done with $\mathrm{MC}$ event generators. Figure 3 shows that $k_{t^{-}}$ algorithm jet resolution parameters, for example $y_{3, g}$, are essentially not affected by hadroni-
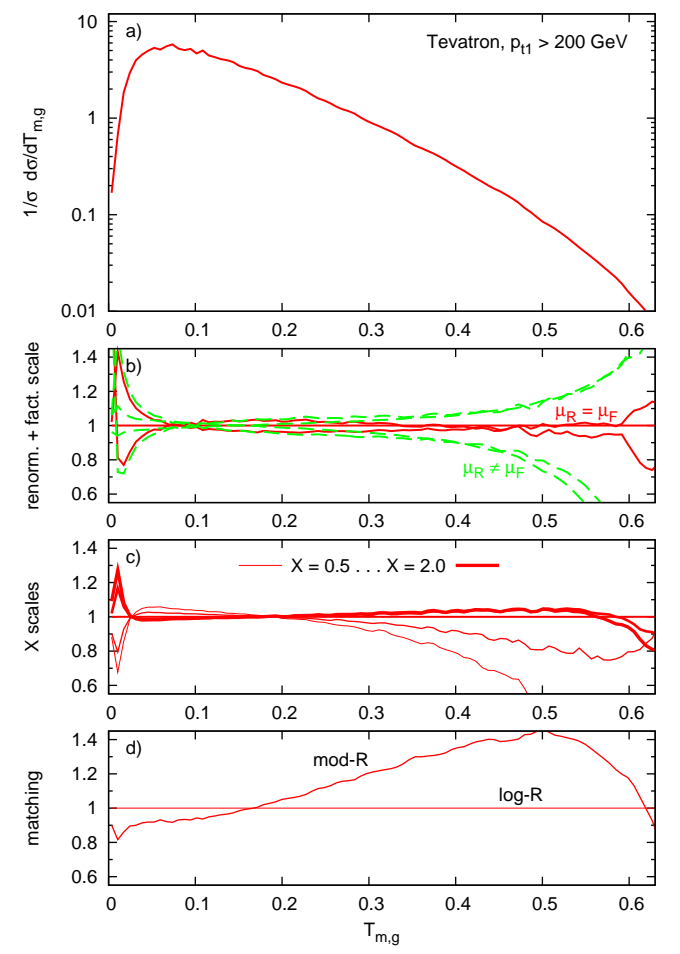

Figure 2: NLL+NLO predictions and theoretical uncertainties for the distribution in $T_{m, g}$. sation and underlying event, while event shapes,

like $\rho_{T, \mathscr{E}}$, get moderate hadronisation corrections, falling as an inverse power of the jet $p_{t}$, but get a huge contribution from the underlying event. This different sensitivity shows that event-shape distributions can be exploited for the validation of MC event generators. Jet resolution parameters are better suited for tunings of parton shower parameters, while with remaining event shapes one can test models of hadronisation and underlying event.
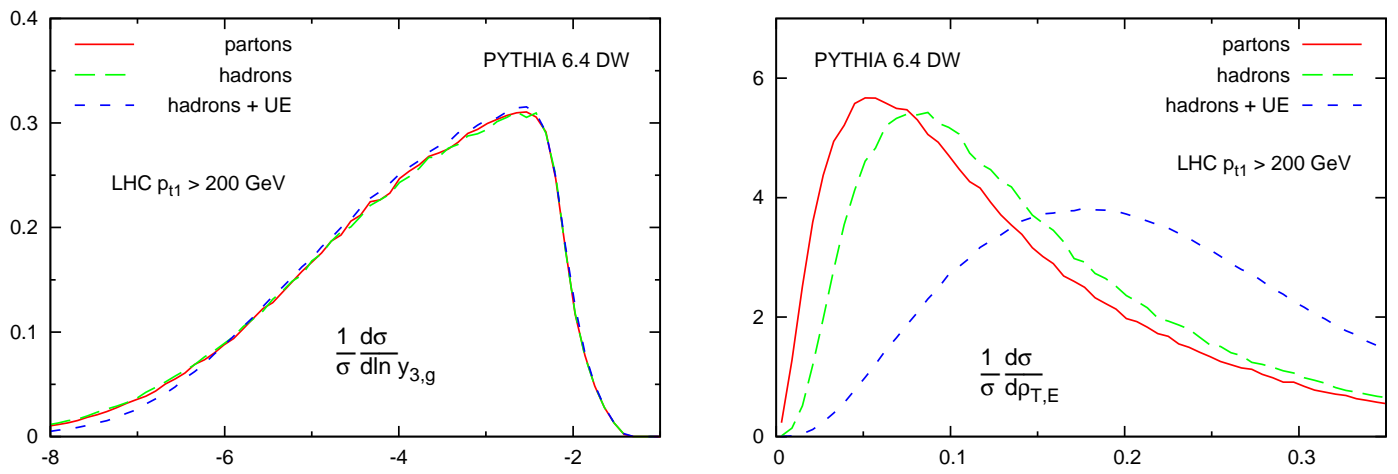

Figure 3: Distributions in directly global three-jet resolution $y_{3, g}$ (left) and exponentially suppressed total mass $\rho_{T, \mathscr{E}}$ (right), as obtained with the MC event generator PYTHIA [8] for LHC with $\sqrt{s}=14 \mathrm{TeV}$. 


\section{Event shapes for New Physics}

A common use of event shapes is that of discriminating among events with different topologies. This is particularly important in New Physics searches, where one expects events with massive particles to be much broader than dijet events. We have then tried to assess the performance of known hadronic event shapes for such studies. First, when considering symmetric events with an arbitrary number of particles in the transverse plane, one can only distinguish between two- and multi-jet events, irrespectively of the number of jets. One can then try to discriminate among different topologies in a sample with the same number of jets (three jets in the considered case). One then finds (see figure 4) that infrared and collinear safe variables fare much better in this respect than unsafe ones (like the widely used transverse sphericity). We remark also that event shapes like
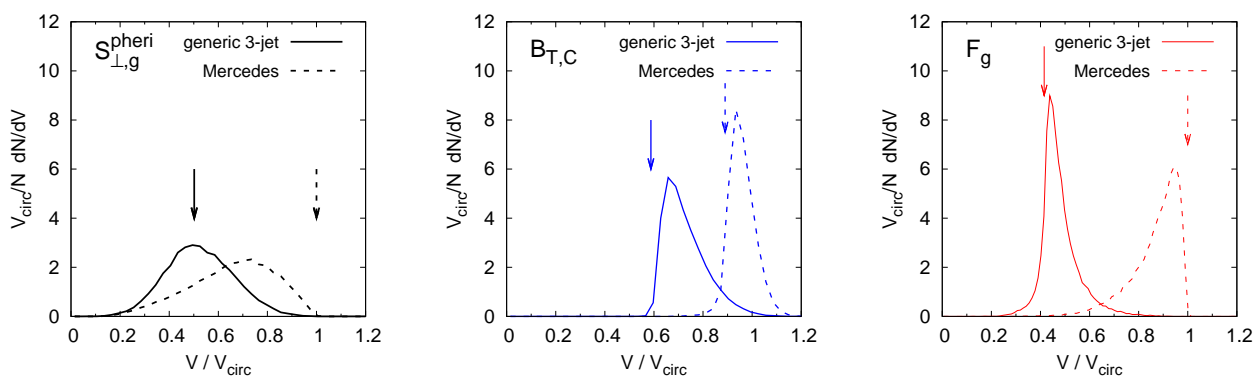

Figure 4: Distribution in the value of three different variables obtained from events with two different hard configurations dressed with parton shower. The two starting configurations are a generic three-parton and a totally symmetric (Mercedes) event in the transverse plane.

the broadenings, which treat tranverse and longitudinal degrees of freedom on equal footing, are better suited for the identification of massive particle decays, since their value is hardly affected by the orientation of the event plane.

For practical applications, it is however desirable to have variables that are more sensitive to the spherical limit. One example presented in [5] is the supersphero observable which is non-zero only for events in which there are three non-coplanar particles in each of the "hemispheres" in which the event is divided by the transverse thrust axis. We believe that phenomenological applications of variables like supersphero, as well as better final-state observables for New Physics, constitute an important subject that deserves further studies.

\section{References}

[1] M. Dasgupta and G. P. Salam, J. Phys. G 30 (2004) R143 [arXiv:hep-ph/0312283].

[2] F. Abe et al. [CDF Collaboration], Phys. Rev. D 44 (1991) 601.

[3] I. A. Bertram [D0 Collaboration], Acta Phys. Polon. B 33 (2002) 3141.

[4] A. Banfi, G. P. Salam and G. Zanderighi, JHEP 0408 (2004) 062 [arXiv:hep-ph/0407287].

[5] A. Banfi, G. P. Salam and G. Zanderighi, JHEP 1006 (2010) 038 [arXiv:1001.4082 [hep-ph]].

[6] Z. Nagy, Phys. Rev. D 68 (2003) 094002 [arXiv:hep-ph/0307268].

[7] A. Banfi, G. P. Salam and G. Zanderighi, JHEP 0503 (2005) 073 [arXiv:hep-ph/0407286].

[8] T. Sjostrand, S. Mrenna and P. Skands, JHEP 0605 (2006) 026 [arXiv:hep-ph/0603175]. 